\title{
Propiedades psicométricas de la Escala de Bienestar Psicológico para Adolescentes (BIEPS-J) en una muestra mexicana
}

\author{
David Luna ${ }^{1}$, Rosa Paola Figuerola-Escoto ${ }^{2}$, José Contreras-Ramírez ${ }^{3}$, Juan José Luis \\ Sienra-Monge ${ }^{4}$, Elsy Maureen Navarrete-Rodríguez ${ }^{5}$, Juana Serret Montoya ${ }^{6}$, Patricia \\ Castañeda Peña ${ }^{7}$ y Fernando Meneses-González ${ }^{8}$
}

\begin{abstract}
RESUMEN
El bienestar psicológico se refiere a la satisfacción general que los individuos tienen respecto de su vida. Este concepto se ha relacionado con la salud mental y con la detección de factores favorables al desarrollo humano. El interés en su estudio en adolescentes se ha incrementado en los últimos años debido a los cambios físicos, mentales y sociales propios a esta etapa del desarrollo, por lo que son necesarios instrumentos válidos y confiables que permitan su evaluación. El objetivo de este estudio fue evaluar propiedades psicométricas de la Escala de Bienestar Psicológico para Adolescentes (BIEPS-J) en una muestra mexicana de adolescentes tardíos y obtener evidencia de su validez convergente y de criterio. Se aplicó la escala BIEPS-J y la Escala de Autoestima de Rosenberg (EAR) a 271 estudiantes de ambos sexos de entre 16 y 18 años. Un análisis paralelo indicó dos factores presentes en la escala BIEPS-J, estructurados mediante un análisis factorial exploratorio (componentes principales, rotación oblicua) y que explicaron el $54 \%$ de la varianza. Los factores fueron: 1) Relación con uno mismo y con terceros, satisfacción con sus relaciones sociales y la aceptación de sí mismo; 2) Planes y recursos personales de control, sensación del dominio de su entorno y auto-competencia, además de la presencia de metas en su vida. La consistencia interna global $(\mathrm{a}>\mathrm{.80})$ y por factor $(\mathrm{a}>\mathrm{.70})$ fue aceptable. La escala BIEPS-J mostró validez convergente con la EAR $(r=.44)$ y el nivel de bienestar psicológico se asoció al nivel de autoestima $(p<.001)$. El puntaje total y por factor de la escala BIEPS-J no difirió entre sexos $(p>$.05). Los datos indican que la escala BIEPS-A es un instrumento válido y confiable para evaluar el bienestar psicológico en población adolescente mexicana de entre 16 y 18 años.
\end{abstract}

Palabras clave: bienestar psicológico, adolescentes, validación, consistencia interna, México.

\footnotetext{
${ }^{1}$ Comisión Nacional de Arbitraje Médico, México; dluna@conamed.gob.mx; https://orcid.org/0000-00030427-3789

2 Instituto Politécnico Nacional, México; rfiguerolae@ipn.mx; https://orcid.org/0000-0002-1349-4623

3 Universidad Nacional Autónoma de México, Escuela Nacional Preparatoria 3 "Justo Sierra", México; pepecapotondi@gmail.com; https://orcid.org/0000-0002-1909-9368

4 Hospital Infantil de México Federico Gómez, México; jjsienra@hotmail.com; https://orcid.org/0000-00015712-052X

${ }^{5}$ Hospital Infantil de México Federico Gómez, México; draenavarrete@gmail.com; https://orcid.org/00000001-9876-3206

6 Hospital Infantil de México Federico Gómez, México; juana.serret07@gmail.com; https://orcid.org/00000002-9426-540X

7 Hospital Infantil de México Federico Gómez, México; picp4474@hotmail.com; https://orcid.org/00000002-3456-8126

8 Comisión Nacional de Arbitraje Médico, México; fmenesesg@conamed.gob.mx; https://orcid.org/00000002-3833-8467
} 
Luna, D., Figuerola-Escoto, R. P., Contreras-Ramírez, J., Luis Sienra-Monge, J. J., Navarrete-Rodríguez, E. M., Serret Montoya, J., Castañeda Peña, P. \& Meneses-González, F. / Psicodebate, $20(1), 43$ - 55.

\title{
Psychometric properties of the Psychological Wellness Scale for Adolescents (BIEPS-J) in a Mexican sample
}

\begin{abstract}
Psychological well-being refers to the satisfaction that individuals generally have regarding their life. This concept has been related to mental health and to the detection of factors that favor human development. The interest in their study in adolescents has increased in recent years due to physical, mental and social changes at this stage of development, so it is necessary valid and reliable instruments that allow their evaluation. The objective of this study was to evaluate psychometric properties of the Psychological Wellbeing Scale for Adolescents (BIEPS-J) in a Mexican sample of late adolescents and obtain evidence of their convergent and criterion validity. The BIEPS-J scale and the Rosenberg Self-esteem Scale (RSS) were applied to 271 students of both sexes between 16 and 18 years old. A parallel analysis indicated two factors present in the BIEPS-J scale, which were structured by exploratory factor analysis (main components, oblique rotation) and that explained $54 \%$ of the variance. The factors were: 1) Relationship with self and with others, indicates satisfaction with their social relationships and self-acceptance; 2) Plans and personal control resources, indicates the sensation of the domain of their environment and self-competence as the presence of goals in their life. The global internal consistency $(a>.80)$ and by factor $(a>.70)$ was acceptable. The BIEPS-J scale showed convergent validity with the RSS $(r=.44)$ and the level of psychological well-being was associated with the level of self-esteem $(p<.001)$. The total score and by factor of the BIEPS-J scale did not differ between sexes $(p>$.05). The data indicate that the BIEPS-A scale is a valid and reliable instrument to assess psychological well-being in the Mexican adolescent population between 16 and 18 years of age.

Keywords: psychological well-being, adolescents, validation, internal consistency, Mexico.
\end{abstract}

La adolescencia es una fase de transición entre la niñez y la adultez, dividida en adolescencia temprana y tardia, que abarcan de los 10 a los 14, y de los 15 a los 19 años, respectivamente (Organización Mundial de la Salud, 2019). Se caracteriza por cambios fisiológicos y físicos (Crone \& Dahl, 2012), que a su vez causan e influyen en cambios cognitivos y psicosociales (Sanders, 2013). Es también un periodo con alta tasa de eventos adversos al desarrollo (Ernst \& Fudge, 2009; Somerville, Jones, \& Casey, 2010; Steinberg, 2008), por lo que, a partir de un enfoque patogénico para su estudio, la han considerado como una etapa de crisis (e.g., Sharp \& Wall, 2018). No obstante, la adolescencia es también una instancia en el que se definen aspiraciones personales y metas a largo plazo (Crone \& Dahl, 2012). En consecuencia, es necesario no solo estudiar su patología sino también los factores que potencian el desarrollo saludable de los adolescentes, tanto a nivel físico como mental, a fin de que estos puedan contar con los recursos necesarios para alcanzar las metas y aspiraciones propuestas (Lacunza \& Contini, 2016).

\section{El enfoque salutogénico en el abordaje de la salud}

En años recientes, el paradigma centrado en el estudio de la patogenia se ha desplazado hacia uno enfocado en el estudio de factores psicosociales promotores de salud física y mental (Domínguez, 2014). Este cambio es coherente con el concepto enunciado para salud (Organización Mundial de la Salud, 1948) y salud mental (Organización Mundial de la Salud, 2001) por la Organización Mundial de la Salud, los cuales incorporan aspectos positivos en el individuo tales como su bienestar, su capacidad para afrontar situaciones adversas y contribuir a la sociedad. Este cambio es también consistente con la 
Carta de Ottawa para la Promoción de la Salud (Organización Mundial de la Salud, 1986), la cual implica el proceso por el cual un individuo toma un papel activo en el resguardo y promoción de su salud.

El enfoque salutogénico es acorde a esta visión positiva de la salud y se ha sugerido su empleo como instrumento para su promoción (Talabera, Salgado, Hernández, \& Borroto, 2014). Este enfoque se centra en el estudio de variables psicológicas que permiten a los individuos afrontar estados de estrés y otros problemas de naturaleza física y psicológica, las que son protectoras ante el riesgo de enfermedad (Rodríguez, Couto, \& Díaz, 2016). Una de estas variables es el bienestar (González, 2004).

\section{Bienestar, bienestar psicológico, y bienestar psicológico en la adolescencia}

El estudio científico del bienestar se ha conducido desde la perspectiva hedónica y la eudemónica (Kaur \& Poja, 2016), si bien en años recientes se ha desarrollado un tercer enfoque que integra ambas posturas (Hone, Jarden, Schofield, \& Duncan, 2014). Desde la perspectiva hedónica, el bienestar subjetivo implica los afectos positivos percibidos por los eventos ocurridos en la vida (Diener, 1984). En cambio, desde la perspectiva hedónica, el bienestar psicológico está centrado en la auto-realización (Ryff, 1989). El bienestar psicológico se ha definido como un estado afectivo positivo que favorece el funcionamiento óptimo en la vida personal y social, permite a los individuos percibir control de su vida y de su entorno, y los motiva a una superación personal mediante acciones pertinentes a ello (Casullo, 2002; Deci \& Ryan, 2008).

El bienestar psicológico es un componente de la salud mental y un factor protector de la salud física, que además promueve procesos de recuperación de la enfermedad, emociones positivas, procesos cognitivos funcionales y el desarrollo social y comunitario (Edwards, 2006; Fredrickson, 2009; Hernández et al., 2018; Huppert, 2009; Vázquez, Hervás, Rahona, \& Gómez, 2009).

La mayor cantidad de investigaciones acerca del bienestar psicológico se han realizado con adultos y estudiantes universitarios. En cambio, su estudio en adolescentes es aún escaso (Khan, Taghdisi, \& Nourijelyani, 2015). Pese a ello, en esta población existe evidencia de diferencias en función del sexo (Kahn et al. 2015; Sagone \& de Caroli, 2014), edad, nivel educativo de los padres y número de miembros en la familia (Kahn et al. 2015). También se ha reportado su asociación al uso de estrategias de afrontamiento adaptativas (Contini, Coronel, Levin, \& Estevez, 2003), al mayor nivel de autoestima (Morales \& González, 2014), felicidad (Heizomi, Allahverdipour, Jafarabadi, \& Safaian, 2015), sentido de vida (Rathi \& Rastogi 2007), valentia, perseverancia, gratitud, inteligencia social y autocontrol (García-Álvarez, JoséSoler, \& Achard-Braga, 2017), y autoeficacia general (de Caroli \& Sagone 2014), así como al menor nivel de ansiedad-estado (Álvarez-Díaz, 2014). Es un predictor de resiliencia (Sagone \& de Caroli, 2014) y puede ser instruido mediante intervención psicológica en contextos no clínicos (Ruini et al., 2009).

Por sus efectos benéficos en los adolescentes y en la población en general, se ha afirmado que las acciones para mejorar el bienestar psicológico podrian tener beneficios politicos, sociales, en la salud pública y en la politica y economía de esta última (Diener, Lucas, Schimmack, \& Helliwell, 2009; Enns et al., 2016). 
Luna, D., Figuerola-Escoto, R. P., Contreras-Ramírez, J., Luis Sienra-Monge, J. J., Navarrete-Rodriguez, E. M., Serret Montoya, J., Castañeda Peña, P. \& Meneses-González, F. / Psicodebate, $20(1), 43$ - 55.

\section{Medición del bienestar psicológico: escalas para adultos y su adaptación para adolescentes}

Si bien actualmente no existe un instrumento que sea considerado como un estándar de oro (Winefield, Gill, Taylor, \& Pilkington, 2015) para evaluar el bienestar psicológico, el trabajo de Carol Ryff ha ejercido amplia influencia en la investigación actual al respecto. Ryff (1989) elaboró el modelo multidimensional del bienestar psicológico, en el cual incorporó como sus dimensiones la autoaceptación, relaciones positivas con otras personas, autonomía, dominio del entorno, propósito en la vida y crecimiento personal. Desarrolló también las Escalas de Bienestar Psicológico (EBP), que en su versión original poseen 20 reactivos por dimensión. Sobre la base de versiones reducidas de las EBP, su validación al danés arrojó una versión con un factor de segundo orden que integra a los seis factores originales, cada uno con entre seis y ocho reactivos (van Dierendonck, 2005). Esta última versión fue la base para la validación al castellano de las EBP, cuyos resultados mostraron una versión con estructura semejante a la de van Dierendonck (2005), aunque con todavía un menor número de reactivos por factor (Díaz et al., 2006).

A partir del trabajo de Díaz et al. (2006), se realizaron estudios para adaptar las EBP con población adolescente en países de habla hispana. Los resultados obtenidos mostraron una estructura con cuatro (México: LoeraMalvaez, Balcázar-Nava, Trejo-González, Gurrola-Peña, \& Bonilla-Muñoz, 2008; Argentina: Meier \& Oros, 2019) y seis factores (Chile: Gallardo Cuadra \& Moyano-Díaz, 2012), con un número de reactivos por factor que oscila entre 4 y 15 y una consistencia interna por factor que va de mala a elevada. Pese a las diferencias en estructura, estas validaciones de las EPB han conservado al menos parcialmente las dimensiones y el concepto de bienestar psicológico del modelo de Ryff. Se ha sugerido que las diferencias entre las EBP adaptadas en población adolescente de habla hispana y la versión original pueden deberse a que esta última fue diseñada para adultos de nacionalidad norteamericana (Fernandes, Vasconcelos-Raposo, \& Teixeira, 2010).

Basados en los planteamientos de Ryff (1989), Casullo y Castro Solano (2000; Casullo, 2002) desarrollaron la Escala de Bienestar Psicológico para Adolescentes (BIEPS-J) para evaluar el bienestar psicológico en adolescentes argentinos de entre 13 y 18 años. La versión preliminar incluyó las dimensiones control de situaciones, vinculos psicosociales, proyectos, aceptación de sí mismo, y autonomía, aunque esta última fue descartada en el proceso de validación psicométrica. Los autores argumentaron que este resultado fue esperado, dado que en países de habla hispana los adolescentes tienden a mantener relaciones más estrechas con sus padres (c.f., GonzálezFuentes \& Andrade, 2016). La escala BIEPS-J ha mostrado propiedades psicométricas adecuadas para fungir un indicador global de bienestar psicológico en su población meta (Casullo \& Castro Solano, 2000) y se ha sugerido su utilidad para rastrillajes (screenings), evaluación psicológica individual e investigación (Casullo, 2002). Entre sus ventajas frente a las EBP se encuentra su diseño específico para población adolescente de cultura hispana y ser un instrumento breve que optimiza el tiempo de recolección de información garantizando la calidad de esta.

Actualmente, no hay información acerca de las propiedades psicométricas de la escala BIEPS-J en población adolescente mexicana que permita determinar si es un instrumento útil para evaluar su bienestar psicológico. El objetivo de este estudio fue evaluar propiedades psicométricas 
de la escala BIEPS-J en una muestra mexicana de adolescentes tardíos y obtener evidencia de su validez convergente y de criterio.

\section{Método}

\section{Participantes}

Mediante un muestreo no probabilístico por conveniencia se reclutó a 271 estudiantes del nivel medio superior de una escuela pública. Fueron 153 mujeres y 118 hombres, de entre 16 y 18 años $(M=16.52, D E=.69)$. El tamaño de la muestra satisfizo los criterios para realizar análisis factorial exploratorio, con 20 participantes por reactivo del instrumento a validar (DeVon et al., 2007).

\section{Instrumentos}

Escala de Bienestar Psicológico para Adolescentes (BIEPS-J; Casullo \& Castro Solano, 2000). Comprende 13 reactivos con tres opciones de respuesta con un puntaje de 1 (En desacuerdo) a 3 (De acuerdo). Se compone de cuatro factores, control: sensación de dominio del entorno y autocompetencia (cuatro reactivos; $a=.56$ ); vínculos: capacidad para establecer adecuadas relaciones sociales (tres reactivos; $a=.51$ ); proyectos: presencia de objetivos y metas en la vida (tres reactivos; $a=.55$ ); aceptación: conformidad con los aspectos buenos y malos de uno mismo (tres reactivos; $a=.50$ ). Explica el $51 \%$ de la varianza y tiene un a de Cronbach global $=.74$. Se ha argumentado que el bajo nivel de consistencia interna de sus factores es debido al reducido número de reactivos en cada uno y no a una falta de homogeneidad en ellos. Un puntaje total menor al percentil 25 indica un bajo nivel de bienestar psicológico, mientras que uno igual o mayor al percentil 50 y 75 indica un nivel promedio y alto respectivamente (Casullo, 2002).

Escala de Autoestima de Rosenberg (EAR; Rosenberg, 1965), validada con población mexicana (Jurado, Jurado, López, \& Querevalú, 2015). Comprende ocho reactivos con cuatro opciones de respuesta con un puntaje de 1 (Muy en desacuerdo) a 4 (Muy de acuerdo). Explica el 36\% de la varianza y tiene un a de Cronbach $=.79$. De acuerdo con el puntaje obtenido, el nivel de autoestima se considera bajo (1-25), leve (26-28), alto (29-30), y muy alto (31-32).

\section{Diseño}

Estudio instrumental (Montero \& León, 2007). La validez de constructo de la escala BIEPS-J se evaluó mediante análisis factorial exploratorio, previa realización de un análisis paralelo. La consistencia interna se evaluó por a de Cronbach y a ordinal. Se identificó la validez convergente de la escala BIEPS-J con la EAR.

\section{Procedimiento}

Previo a la validación psicométrica, se condujo una adaptación cultural. Participaron 30 estudiantes del nivel medio superior de una escuela pública, diferentes a los antes descritos. Los estudiantes recibieron un formato con los reactivos de la escala BIEPS-J y se les solicitó indicar si comprendían la idea que cada uno expresaba, además de anotar la o las palabras de difícil comprensión. Debido a que cada reactivo fue comprendido por más del $90 \%$ de los estudiantes, se decidió utilizar la redacción original de la escala BIEPS-J. 
Luna, D., Figuerola-Escoto, R. P., Contreras-Ramírez, J., Luis Sienra-Monge, J. J., Navarrete-Rodríguez, E. M., Serret Montoya, J., Castañeda Peña, P. \& Meneses-González, F. / Psicodebate, $20(1), 43$ - 55.

Para la validación psicométrica, durante la hora de clase un investigador solicitó a los estudiantes su colaboración voluntaria en el estudio, explicó sus objetivos y aclaró dudas. Los estudiantes que aceptaron participar recibieron una bateria impresa con un consentimiento informado que sus padres debian firmar, una carta de asentimiento que ellos debian firmar y los instrumentos impresos. Se solicitó la entrega de la batería al día siguiente de su recepción.

\section{Análisis de datos}

Para cada reactivo se calculó la media, desviación estándar, coeficiente de sesgo y curtosis. Se evaluó la normalidad univariada con la prueba de Shapiro-Wilk y la multivariada con el cálculo del coeficiente de Mardia. La capacidad de discriminación de los reactivos se estimó mediante la estrategia de grupos extremos, comparando con pruebas $t$ de una cola para grupos independientes los puntajes menores y mayores al primer y tercer cuartil de cada reactivo. Se empleó esta prueba por ser robusta y soportar desviaciones de la normalidad (de Winter, 2013). Se calculó la correlación reactivo-total corregida. Los reactivos sin capacidad discriminativa o con una correlación reactivo-total corregida $r<.20$ fueron eliminados (Cortada de Kohan, 2004).

La adecuación muestral de los datos se verificó mediante el cálculo de la determinante de la matriz de correlación, la prueba de Bartlett y el índice KMO. Se realizó un análisis paralelo y se retuvo aquellos factores cuya varianza explicada rebasó el percentil 95 de la varianza explicada por factores conformados al azar. Por sus características, el análisis paralelo es considerado uno de los procedimientos más precisos para identificar el número de factores a retener cuando se realiza análisis factorial (Hayton, Allen, \& Scarpello 2004), además de poseer un carácter objetivo en contraste con el generalmente empleado criterio de Kaiser y el análisis del gráfico de sedimentación con los cuales se tiende a sobreestimar el número de factores (Baglin, 2014). Después de identificar el número de factores a retener, se condujo un análisis factorial exploratorio a partir de la matriz de correlaciones policóricas, empleando el método de componentes principales y rotación oblicua promax (Huamani \& Arias, 2018). La conformación de los factores requirió al menos tres reactivos por factor, cada reactivo con saturación $\geq .40$ en un solo factor (estructura factorial simple) y una comunalidad $\left(h^{2}\right) \geq .32$ (Tabachnick \& Fidell, 1996), la congruencia conceptual reactivo-factor y que cada factor mostrara una confiabilidad $\geq .70$ calculada por el a de Cronbach y el a ordinal. Este último se empleó debido a que es un indicador más adecuado de consistencia interna cuando se tienen escalas ordinales con cinco o menos opciones de respuesta, además de que existe evidencia de que el a de Cronbach tiende a subestimar el valor de esta medida (Elosúa-Oliden \& Zumbo, 2008). El a de Cronbach y el a ordinal se emplearon también para calcular la consistencia interna global y se consideró aceptable un valor $\geq .70$ (George \& Mallery, 2003).

La estructura obtenida con el análisis previo y sus propiedades fue comparada con una estructura calculada ex profeso para arrojar cuatro factores, como la originalmente reportada por Casullo y Castro Solano (2000). Esto, para evaluar la pertinencia teórica y psicométrica de ambas.

La validez convergente se evaluó mediante la correlación productomomento de Pearson entre el puntaje de la EAR y de la escala BIEPS-J y sus factores. La fuerza de asociación entre variables se interpretó trivial con 
valores absolutos menores a .10 , baja .11 a .29 , media .30 a .49 , y alta $\geq .50$ (Ellis, 2010).

La validez de criterio de la escala BIEPS-J se evaluó mediante la contrastación entre grupos. Con pruebas $x^{2}$ de independencia a una cola se estimó la asociación entre el nivel de bienestar psicológico y el nivel de autoestima. Con un resultado significativo se calcularon los residuos estandarizados de Pearson como prueba post hoc y la $V$ de Cramer como indicador de la fuerza de asociación entre variables. Esta última se interpretó siguiendo a Ellis (2010). Con pruebas $t$ de dos colas para grupos independientes se comparó el puntaje total y por factor de la escala BIEPS-J entre sexos. Se empleó la $d$ de Cohen como índice del tamaño del efecto, y se consideró un efecto pequeño, mediano y grande, una $d \geq .20, .50, .80$ respectivamente (Aron \& Aron, 2001).

Para el análisis de datos se empleó el programa FACTOR v.10.8.04 y el SPSS v.20.

\section{RESULTADOS}

\section{Análisis descriptivo de los reactivos y capacidad discriminativa}

La tabla 1 muestra los estadísticos descriptivos por reactivo. La media y la desviación estándar se encontraron en un rango de 2.26 a 2.74 y de .52 a .73 respectivamente. No hubo evidencia de normalidad univariada $(p<.001)$ o multivariada (coeficiente de asimetria $=28.22, p=1$; coeficiente de curtosis $=$ $220.11, p<.001)$. Todos los reactivos discriminaron $(p<.001)$ y mostraron una correlación reactivo-total corregida $r>.20$.

\section{Estructura factorial y consistencia interna}

Las pruebas de adecuación muestral indicaron una determinante de matriz de correlación $=.016$, un indice $\mathrm{KMO}=.82$, y una prueba de esfericidad de Bartlett $=1099, p<.001$. Esto indicó la pertinencia de los datos para realizar un análisis factorial. El análisis paralelo reveló la presencia de dos factores cuya varianza explicada rebasó el percentil 95 de la varianza explicada por factores aleatorios. Así, retener un número mayor de factores no agregaria información relevante a la validación del BIEPS-J en la población meta. La tabla 1 muestra los resultados del análisis factorial exploratorio, el cual reveló una estructura simple: reactivos asignados a un solo factor y con cargas mayores a .40. La excepción fue el reactivo 4 que tuvo una comunalidad y una carga factorial inaceptable. Los dos factores retenidos estuvieron conformados por la conjunción de los factores vinculos/aceptación, y control/proyectos de la estructura original del BIEPS-J, y en su conjunto explicaron el $54 \%$ de la varianza, mostrando una correlación positiva entre ambos. Las comunalidades $\left(h^{2}\right)$ demostraron la pertinencia del uso del análisis de componentes principales para la extracción de factores. La consistencia interna por factor (tabla 1) y global, a de Cronbach $=.80 \mathrm{y}$ a ordinal $=.92$, estuvo en el rango de aceptable a elevada. 
Luna, D., Figuerola-Escoto, R. P., Contreras-Ramírez, J., Luis Sienra-Monge, J. J., Navarrete-Rodríguez, E. M., Serret Montoya, J., Castañeda Peña, P. \& Meneses-González, F. / Psicodebate, $20(1), 43$ - 55.

Tabla 1.

Análisis de los reactivos, estructura factorial, y validez convergente de la escala de Bienestar Psicológico para Adolescentes (BIEPS-J) en una muestra mexicana.

\begin{tabular}{|c|c|c|c|c|c|c|c|c|}
\hline REACTIVO & $M$ & $D E$ & $s$ & $C$ & F1 & F2 & $r_{(r-t c)}$ & $h^{2}$ \\
\hline \multirow{2}{*}{$\begin{array}{l}7 \text { Generalmente le caigo bien a la gente } \\
\text { Creo que en general me llevo bien con } \\
11 \text { la gente }\end{array}$} & 2.35 & 0.59 & -.28 & -.67 & .89 & & .38 & .69 \\
\hline & 2.54 & 0.56 & -.75 & -.46 & .87 & & .42 & .56 \\
\hline $\begin{array}{l}8 \text { Cuento con personas que me ayudan } \\
\text { si lo necesito }\end{array}$ & 2.74 & 0.52 & -1.95 & 2.98 & .76 & & .42 & .62 \\
\hline \multirow{2}{*}{$\begin{array}{l}2 \text { Tengo amigos/as en quien confiar } \\
9 \text { Estoy bastante conforme con mi forma } \\
\text { de ser }\end{array}$} & 2.68 & 0.56 & -1.61 & 1.62 & .70 & & .40 & .55 \\
\hline & 2.42 & 0.65 & -.69 & -.54 & .45 & & .53 & .53 \\
\hline $\begin{array}{l}13 \text { Puedo aceptar mis equivocaciones y } \\
\text { tratar de mejorar }\end{array}$ & 2.68 & 0.53 & 1.43 & 1.13 & & .88 & .54 & .74 \\
\hline $\begin{array}{l}5 \text { Si algo me sale mal puedo aceptarlo, } \\
\text { admitirlo }\end{array}$ & 2.60 & 0.57 & -1.16 & .36 & & .87 & .45 & .65 \\
\hline $\begin{array}{l}\text { Si estoy molesto/a por algo soy capaz } \\
\text { de pensar en cómo cambiarlo }\end{array}$ & 2.40 & 0.62 & -.55 & -.60 & & .81 & .42 & .56 \\
\hline $\begin{array}{l}\text { Soy una persona capaz de pensar un } \\
\text { proyecto para mi vida }\end{array}$ & 2.56 & 0.59 & -1.05 & .10 & & .70 & .48 & .54 \\
\hline $\begin{array}{l}1 \text { Creo que me hago cargo de lo que } \\
\text { digo o hago }\end{array}$ & 2.60 & 0.57 & -1.18 & .41 & & .64 & .45 & .49 \\
\hline $\begin{array}{l}\text { Creo que sé lo que quiero hacer con } \\
\text { mi vida }\end{array}$ & 2.38 & 0.68 & -.66 & -.68 & & .53 & .41 & .36 \\
\hline \multirow{2}{*}{$\begin{array}{l}6 \begin{array}{l}\text { Me importa pensar qué haré en el } \\
\text { futuro }\end{array} \\
4 \begin{array}{l}\text { En general estoy conforme con el } \\
\text { cuerpo que tengo }\end{array}\end{array}$} & 2.73 & 0.50 & -1.76 & 2.29 & & .51 & .40 & .43 \\
\hline & 2.26 & 0.73 & -.45 & -1.04 & & .35 & .35 & .26 \\
\hline \multicolumn{9}{|c|}{ Propiedades de los factores } \\
\hline \multirow{3}{*}{\multicolumn{5}{|c|}{$\begin{array}{r}\text { Autovalor } \\
\% \text { Varianza explicada } \\
\text { a de Cronbach }\end{array}$}} & 5.55 & 1.62 & & \\
\hline & & & & & 42 & 12 & & \\
\hline & & & & & $\begin{array}{r}.71 \\
85\end{array}$ & $\begin{array}{r}.75 \\
87\end{array}$ & & \\
\hline \multicolumn{5}{|c|}{$\alpha$ ordinal } & & & & \\
\hline \multicolumn{9}{|c|}{ Correlación entre factores } \\
\hline \multirow{2}{*}{\multicolumn{5}{|c|}{$\begin{array}{l}\text { F1: Vínculos/aceptación } \\
\text { F2: Control/proyectos }\end{array}$}} & 1 & & & \\
\hline & & & & & .50 & 1 & & \\
\hline \multicolumn{9}{|c|}{ Correlación con Escala de Autoestima de Rosenberg (EAR) } \\
\hline & & & & I EAR & $.44^{* *}$ & $.31^{* *}$ & & \\
\hline
\end{tabular}

Nota: $M=$ media; $D E$ = desviación estándar; $S=$ sesgo; $C=$ curtosis; $F=$ Factor; $r_{(r e a c-T c)}=$ correlación reactivo-total corregida $h^{2}=$ comunalidad; ${ }^{* *} p<.001$

\section{Comparación de la estructura factorial con dos y cuatro factores para la escala BIEPS-J}

Las propiedades psicométricas de la escala BIEPS-J con dos y cuatro factores se muestran en la tabla 2. Esta última estructura resultó insatisfactoria puesto que el análisis paralelo indicó que los factores tres y cuatro no explican más varianza que cualquier otro factor conformado al azar. Además, los factores dos y tres mostraron un alfa de Cronbach insatisfactorio y el Factor cuatro estuvo integrado por dos reactivos, opuesto a los criterios antes descritos de retención de factores. 
Tabla 2.

Propiedades psicométricas de la escala de Bienestar Psicológico para Adolescentes (BIEPS-J) en una muestra mexicana conformada por dos y cuatro factores

\begin{tabular}{|c|c|c|c|c|c|c|c|c|c|c|}
\hline \multirow{2}{*}{ Estructura } & \multicolumn{4}{|c|}{ Autovalores } & \multicolumn{5}{|c|}{$\alpha$ Cronbach ( $\alpha$ ordinal) } & \multirow[t]{2}{*}{$\%$ VE } \\
\hline & F1 & F2 & F3 & F4 & F1 & F2 & F3 & F4 & Global & \\
\hline Bifactorial & $5.55^{*}$ & $1.62^{*}$ & -- & -- & $.71(.85)$ & $.75(.87)$ & -- & -- & $.80(.92)$ & 54 \\
\hline Tetrafactorial & $4.54^{*}$ & $1.43^{*}$ & 1.13 & 1.04 & $.73(.76)$ & $.65(.77)$ & $.63(.72)$ & $.72(.74)$ & $.80(.92)$ & 62 \\
\hline
\end{tabular}

\section{Validez convergente y validez de criterio}

Se detectó una correlación positiva entre la EAR y la escala BIEPS-A ( $r=$ $.44, p<.001$ ) y sus factores (tabla 1), con una fuerza de asociación entre variables media. Igualmente, hubo una asociación de intensidad baja entre el nivel de autoestima y el nivel de bienestar psicológico $\left(\mathrm{x}^{2}=36.44, p<.001, V=\right.$ .25). El bajo nivel de bienestar psicológico se asoció a bajo nivel de autoestima, mientras que el alto nivel de bienestar psicológico se asoció a leve, alto y muy alto nivel de autoestima. No se detectaron diferencias entre sexos en el puntaje total o por factor del BIEPS-J (tabla 3).

Tabla 3.

Puntaje total y por factor, y comparación entre sexos en la escala de Bienestar Psicológico para Adolescentes (BIEPS-J) en una muestra mexicana.

\begin{tabular}{lcccccc}
\hline & Mín-Máx & $\begin{array}{c}\text { Muestra total } \\
(\boldsymbol{n}=\mathbf{2 7 1}) \\
\boldsymbol{M}(\boldsymbol{D E})\end{array}$ & $\begin{array}{c}\text { Mujeres } \\
(\boldsymbol{n}=\mathbf{1 5 3}) \\
\boldsymbol{M ( D E )}\end{array}$ & $\begin{array}{c}\text { Hombres } \\
(\boldsymbol{n}=\mathbf{1 1 8}) \\
\boldsymbol{M ( D E )}\end{array}$ & $\boldsymbol{t}$ & $\boldsymbol{p}$ \\
\cline { 2 - 7 } & $12-36$ & $30.74(3.92)$ & $31(3.65)$ & $30.40(4.25)$ & 1.24 & .21 \\
Total BIEPS-J & $5-15$ & $12.75(1.97)$ & $12.90(1.79)$ & $12.55(2.18)$ & 1.47 & .14 \\
Vínculos/aceptación & $7-21$ & $17.99(2.62)$ & $18.09(2.44)$ & $17.85(2.84)$ & 0.75 & .45 \\
Control/proyectos & 7.95 &
\end{tabular}

Nota: Mín-Máx = Puntaje mínimo y máximo posible; $M=$ Media; $D E=$ Desviación estándar

\section{COMENTARIOS}

La validación de la escala BIEPS-J con adolescentes tardíos mexicanos indicó que esta posee una estructura bifactorial, con factores correlacionados, una consistencia interna global y por factor en el rango de aceptable a elevada y explicó más del $50 \%$ de la varianza. Mostró la pérdida de un reactivo y los reactivos retenidos presentaron poder discriminativo y una correlación reactivo-total corregida aceptable. Esta versión bifactorial de la escala BIEPS-J presentó validez convergente con la autoestima evaluada mediante la EAR, resultado que es acorde a la literatura (Morales \& González, 2014). Consistente también con los datos obtenidos por Casullo y Castro Solano (2000) y Casullo (2002), no se identificaron diferencias entre sexos en el puntaje total o por factor. Si bien otros estudios han demostrado su ocurrencia (e.g., Kahn et al. 2015; Sagone \& de Caroli, 2014), en ellos se emplearon diferentes instrumentos, con los cuales dicha diferencia ha sido reportada (i.e., EBP: Ryff, 1989; WHO-5: Gómez-Baya, Lucia-Casademunt, \& Salinas-Pérez, 2018).

En contraste con lo antes mencionado, la estructura original con cuatro factores de la escala BIEPS-J no resultó satisfactoria. Esto, principalmente, 
Luna, D., Figuerola-Escoto, R. P., Contreras-Ramírez, J., Luis Sienra-Monge, J. J., Navarrete-Rodríguez, E. M., Serret Montoya, J., Castañeda Peña, P. \& Meneses-González, F. / Psicodebate, $20(1), 43$ - 55.

debido a que solo dos factores explicaron mayor porcentaje de varianza que factores conformados aleatoriamente.

E1 porcentaje de varianza explicada es semejante entre la escala BIEPS$\mathrm{J}$ original y la aquí validada. No obstante, esta última se conformó solo de dos factores. El Factor 1 integró los reactivos de los factores originales Vínculos y Aceptación (excepto por el reactivo 4), e indica que las relaciones sociales que el adolescente mantiene están ligadas al grado en que él mismo se acepta como es. Esta relación ha sido reportada previamente (i.e., Alonso Palacio et al., 2007; Loera et al., 2008). El Factor 2 integró los reactivos de los factores originales Control y Proyectos, e indica que las metas que el adolescente se propone están asociadas con el control que este percibe de su entorno y de la capacidad que, asume, tiene para alcanzarlas. La relación de estos factores es coherente con teorias motivacionales (e.g., Ford, 1992). Esta integración creó entonces dos nuevos factores que pueden dar cuenta del bienestar psicológico en adolescentes de nacionalidad mexicana, siendo el Factor 1 Relación con uno mismo y con terceros y el Factor 2 Planes y recursos personales de control.

En la validación original de la escala BIEPS-J se empleó un análisis factorial exploratorio con rotación ortogonal, por lo que no hay datos acerca de la correlación entre factores (Casullo \& Castro Solano, 2000). En cambio, en la validación aquí presentada la rotación fue oblicua. El resultado fue factores que correlacionan en forma positiva, aunque no a un grado que pueda asumirse la identidad entre ellos. La consistencia interna global y por factor, evaluada por dos métodos distintos, fue también mayor para la validación aquí presentada respecto de la validación original de la escala BIEPS-J. La aceptable consistencia interna de los factores permite hacer una evaluación del bienestar psicológico tanto global como por factor. Esto fortalece la validez de la escala BIEPS-J bifactorial aquí presentada.

Pese a la eliminación de un reactivo, la validación de la escala BIEPS-J aquí mostrada mantuvo su semejanza conceptual con la versión original que, además, fue diseñada y validada expresamente para población hispana de adolescentes. No obstante, $y$ al igual que con las validaciones para adolescentes de las EBP, no fue posible mantener su estructura original. Aunque esto último no necesariamente implica que tanto esta versión bifactorial de la escala BIEPS-J como cada nueva versión de las EBP para adolescentes midan algo distinto al bienestar psicológico, sí dificulta la comparación entre los resultados de cada una y con su respectiva versión original.

Recientemente, González-Fuentes y Andrade (2016) basados también en Ryff (1989), diseñaron y validaron con población mexicana de entre 15 y 20 años la Escala de Bienestar Psicológico para Adolescentes (BP-A). Este instrumento posee 29 reactivos agrupados en siete factores que explican el $60 \%$ de varianza y poseen una consistencia interna por factor de a de Cronbach $=.74$ a .83 y una consistencia global de .76 . Los factores fueron: crecimiento personal, relaciones positivas con otros, propósito de vida, autoaceptación, planes a futuro, rechazo personal y control personal. Si bien hay semejanzas conceptuales entre las escalas BIEPS-J y BP-A, esta última conservó el factor crecimiento personal, del cual se ha resaltado su importancia en el concepto de bienestar psicológico. No obstante, actualmente no hay evidencia sobre las bondades del uso de una frente a la otra escala, por lo que futuros estudios deberán ser realizados al respecto. 
Una de las bondades de este estudio es el empleo del análisis paralelo, el cual ofrece mayor certeza sobre el número de factores que deben ser integrados mediante el análisis factorial (Hayton et al. 2004), además del procedimiento de rotación oblicua empleado. Este último es sugerido, dada la mayor probabilidad de que constructos psicológicos correlacionen entre sí, respecto de un caso opuesto (Thurstone, 1947). Entre las limitaciones de este estudio se encuentran el haber participado únicamente estudiantes. Esto puede ocasionar un problema de generalización a otras poblaciones de adolescentes que no asistan a la escuela y muestren un contexto sociocultural distinto. Además, la escala BIEPS-J debe ser aún sometida a otros tipos de validez como discriminante y predictiva, así como verificar su estructura mediante un análisis factorial confirmatorio. Futuros estudios deberán atender estas limitantes.

\section{CONCLUSIONES}

La escala BIEPS-J validada con población de adolescentes tardíos mexicanos presenta adecuadas propiedades psicométricas para ser empleada como una herramienta de detección del bienestar psicológico en la población señalada. Además, el uso de este instrumento podria representar una estrategia o medio para la promoción y difusión del enfoque salutogénico en la investigación actual con adolescentes mexicanos.

\section{REFERENCIAS}

Alonso-Palacio, L. M., Murcia-Gandara, G., Murcia.Gandara, J., Herrera-Pertuz, D., Gómez-Guzmán, D., Comas-Vargas, M., \& Ariza-Theran, P. (2007). Autoestima y relaciones interpersonales en jóvenes estudiantes de primer semestre de la División Salud de la Universidad del Norte, Barranquilla (Colombia). Salud uninorte, 23(1), 34-44.

Álvarez-Díaz, Y. (2014). Relación del bienestar psicológico con los niveles de ansiedad: rasgo-estado en adolescentes. Medimay, 2O(1), 89-99.

Aron, A. \& Aron, E. (2001). Estadística para psicólogos. Argentina: Prentice Hall.

Baglin, J. (2014). Improving Your Exploratory Factor Analysis for Ordinal Data: A Demonstration Using FACTOR. Practical Assessment, Research \& Evaluation, 19, Article 5.

Casullo, M. (2002). Evaluación del bienestar psicológico en Iberoamérica. Buenos Aires: Paidós.

Casullo, M. M. \& Castro-Solano, A. (2000). Evaluación del bienestar psicológico en estudiantes adolescentes argentinos. Revista de Psicología, 18(1), 35-68.

Contini, N., Coronel, P., Levin, M., \& Estevez, A. (2003). Estrategias de afrontamiento y bienestar psicológico en adolescentes escolarizados de Tucumán. Revista de Psicología, 21(1), 179-200.

Cortada de Kohan, N. (2004). Teoría y métodos para la construcción de escalas de actitudes. Buenos Aires: Lugar Editorial.

Crone, E. A. \& Dahl, R. E. (2012). Understanding adolescence as a period of social-affective engagement and goal flexibility. Nature Reviews Neuroscience, 13(9), 636-650. https://doi.org/10.1038/nrn3313

de Caroli, M. E. \& Sagone, E. (2014). Generalized self-efficacy and well-being in adolescents with high vs. Low scholastic self-efficacy. Procedia-Social and Behavioral Sciences, 141, 867-874. https://doi.org/10.1016/j.sbspro.2014.05.152

de Winter, J. C. (2013). Using the Student's t-test with extremely small sample sizes. Practical Assessment, Research \& Evaluation, 18, 1-12.

Deci, E. L. \& Ryan, R. M. (2008). Hedonia, eudaimonia, and well-being: An introduction. Journal of Happiness Studies, 9, 1-11. https://doi.org/10.1007/s10902-006-9018-1

DeVon, H. A., Block, M. E., Moyle-Wright, P., Ernst, D. M., Hayden, S. J., Lazzara, D. J., ... \& KostasPolston, E. (2007). A psychometric toolbox for testing validity and reliability. Journal of Nursing scholarship, 39(2), 155-164. https://doi.org/10.1111/j.1547-5069.2007.00161.x

Diaz, D., Rodríguez-Carvajal, R., Blanco, A., Moreno-Jiménez, B., Gallardo, I., Valle, C., \& Dierendonck, D. V. (2006). Adaptación española de las escalas de bienestar psicológico de Ryff. Psicothema, 18(3), 572577.

Diener, E. (1984). Subjective well-being. Psychological bulletin, 95(3), 542-575. https://doi.org/10.1037/0033-2909.95.3.542

Diener, E., Lucas, R., Schimmack, U., \& Helliwell, J. (2009). Well-being for public policy. Oxford: Oxford University Press. https://doi.org/10.1093/acprof:oso/9780195334074.001.0001

Dominguez, S. (2014). Análisis psicométrico de la Escala de Bienestar Psicológico para Adultos en estudiantes universitarios de Lima: un enfoque de ecuaciones estructurales. Psychologia, Avances de 
Luna, D., Figuerola-Escoto, R. P., Contreras-Ramírez, J., Luis Sienra-Monge, J. J., Navarrete-Rodríguez, E. M., Serret Montoya, J., Castañeda Peña, P. \& Meneses-González, F. / Psicodebate, $20(1), 43$ - 55.

la Disciplina, 8, 23-31. https://doi.org/10.21500/19002386.1211

Edwards, S. (2006). Physical exercise and psychological well-being. South African Journal of Psychology, 36(2), 357-373. https://doi.org/10.1177/008124630603600209

Ellis, P. D. (2010). The essential guide to effect sizes: An introduction to statistical power, meta-analysis and the interpretation of research results. Cambridge: Cambridge University Press. https://doi.org/10.1017/CBO9780511761676

Elosua-Oliden, P. \& Zumbo, B. D. (2008). Reliability coefficients for ordinal response scales. Psicothema, 20(4), 896-901.

Enns, J., Holmqvist, M., Wener, P., Halas, G., Rothney, J., Schultz, A., .. \& Katz, A. (2016). Mapping interventions that promote mental health in the general population: a scoping review of reviews. Preventive Medicine, 87, 70-80. https://doi.org/10.1016/j.ypmed.2016.02.022

Ernst, M. \& Fudge, J. L. (2009). A developmental neurobiological model of motivated behavior: anatomy, connectivity and ontogeny of the triadic nodes. Neuroscience \& Biobehavioral Reviews, 33(3), 367-382. https://doi.org/10.1016/j.neubiorev.2008.10.009

Fernandes, H. M., Vasconcelos-Raposo, J., \& Teixeira, C. M. (2010). Preliminary analysis of the psychometric properties of Ryff's scales of psychological well-being in Portuguese adolescents. The Spanish Journal of Psychology, 13(2), 1032-1043. https://doi.org/10.1017/S1138741600002675

Ford, M. E. (1992). Motivating humans: Goals, emotions, and personal agency beliefs. Newbyry Park, California: Sage. https://doi.org/10.4135/9781483325361

Fredrickson, B. L. (2009). Positivity: Discover the ratio that tips your life toward flourishing. New York, NY: Crown.

Gallardo-Cuadra, I. \& Moyano-Díaz, E. (2012). Análisis psicométrico de las escalas Ryff (versión española) en una muestra de adolescentes chilenos. Universitas psychologica, 11(3), 940-940. https://doi.org/10.11144/Javeriana.upsy11-3.aper

García-Âlvarez, D., José-Soler, M., \& Achard-Braga, L. (2017). Promoción del bienestar psicológico en la secundaria: una experiencia piloto. Búsqueda, 4(18), 22-35. https://doi.org/10.21892/01239813.335

George, D. \& Mallery, P. (2003). SPSS for Windows step by step: A simple guide and reference. 11.0 update (4th ed.). Boston: Allyn \& Bacon.

Gómez-Baya, D., Lucia-Casademunt, A., \& Salinas-Pérez, J. (2018). Gender differences in psychological well-being and health problems among European health professionals: analysis of psychological basic needs and job satisfaction. International journal of environmental research and public health, 15(7), 1474. https://doi.org/10.3390/ijerph15071474

González-Fuentes, M. B., \& Andrade, P. P. (2016). Escala de Bienestar Psicológico para Adolescentes. Revista Iberoamericana de Diagnóstico y Evaluación-e Avaliação Psicológica, 2(42), 69-83. https://doi.org/10.21865/RIDEP42_69

González, Z. G. C. (2004). La psicología positiva: un cambio en nuestro enfoque patológico clásico. Liberabit, $10,82-88$

Hayton, J. C., Allen, D. G., \& Scarpello, V. (2004). Factor retention decisions in exploratory factor analysis: A tutorial on parallel analysis. Organizational research methods, 7(2), 191-205. https://doi.org/10.1177/1094428104263675

Heizomi, H., Allahverdipour, H., Jafarabadi, M. A., \& Safaian, A. (2015). Happiness and its relation to psychological well-being of adolescents. Asian journal of psychiatry, 16, 55-60. https://doi.org/10.1016/j.ajp.2015.05.037

Hernandez, R., Bassett, S. M., Boughton, S. W., Schuette, S. A., Shiu, E. W., \& Moskowitz, J. T. (2018). Psychological well-being and physical health: Associations, mechanisms, and future directions. Emotion Review, 1O(1), 18-29. https://doi.org/10.1177/1754073917697824

Hone, L. C., Jarden, A., Schofield, G. M., \& Duncan, S. (2014). Measuring flourishing: The impact of operational definitions on the prevalence of high levels of wellbeing. International Journal of Wellbeing, 4(1), 62-90. https://doi.org/10.5502/ijw.v4i1.4

Huamani, C. J. C. \& Arias, G. W. L. (2018). Modelo predictivo del Bienestar Psicológico a partir de la Satisfacción con la Vida en jóvenes de la ciudad de Arequipa (Perú). Revista Latinoamericana de Ciencia Psicológica, 10(2) 1-20.

Huppert, F. A. (2009). Psychological well-being: Evidence regarding its causes and consequences. Applied Psychology: Health and Well-Being, 1(2), 137-164. https://doi.org/10.1111/j.1758-0854.2009.01008.x

Jurado, C. D., Jurado C. S., López V. K., \& Querevalú G. B. (2015). Validez de la Escala de Autoestima de Rosenberg en universitarios de la Ciudad de México. Revista Latinoamericana de Medicina Conductual, 5(1), 18-22.

Kaur, J. M. \& Poja, M. (2016). Relationship between mental health and psychological well-being of prospective female teachers. Journal of Research \& Method in Education, 6, 1-6.

Khan, Y., Taghdisi, M. H. \& Nourijelyani, K. (2015). Psychological Well-Being (PWB) of school adolescents aged 12-18 yr, its correlation with general levels of Physical Activity (PA) and socio-demographic factors in Gilgit, Pakistan. Iranian journal of public health, 44(6), 804-813.

Lacunza, A. B. \& Contini, E. N. (2016). Relaciones interpersonales positivas: los adolescentes como protagonistas. Psicodebate, 16(2), 73-94. https://doi.org/10.18682/pd.v16i2.598

Loera-Malvaez, N., Balcázar-Nava, P., Trejo-González, L., Gurrola-Peña, G. M., \& Bonilla-Muñoz, P. B. (2008). Adaptación de la escala de bienestar psicológico de Ryff en adolescentes preuniversitarios. Revista Neurología, Neurocirugía y Psiquiatría, 41(3-4), 90-97

Meier, L. K. \& Oros, L. B. (2019). Adaptación y Análisis Psicométrico de las Escalas de Bienestar Psicológico de Ryff en Adolescentes Argentinos. Psykhe, 27(2), 1-16. https://doi.org/10.7764/psykhe.27.2.1169

Montero, I. \& León, O. G. (2007). A guide for naming research studies in Psychology. International Journal of Clinical and Health psychology, 7(3), 847-862. 
Morales, M. \& González, A. (2014). Resiliencia-Autoestima-Bienestar psicológico y Capacidad intelectual de estudiantes de cuarto medio de buen rendimiento de liceos vulnerables. Estudios pedagógicos (Valdivia), 4O(1), 215-228. https://doi.org/10.4067/S0718-07052014000100013

Organización Mundial de la Salud (1948). Preamble to the Constitution of the World Health Organization. Recuperado de https://www.loc.gov/law/help/us-treaties/bevans/m-ust000004-0119.pdf

Organización Mundial de la Salud (1986). The Ottawa Charter for Health Promotion. Recuperado de: https://www.who.int/healthpromotion/conferences/previous/ottawa/en/

Organización Mundial de la Salud (2001). The world health report. Mental health: New understanding, new hope. Recuperado de https://www.who.int/whr/2001/en/

Organización Mundial de la Salud (2019). Salud de la madre, el recién nacido, del niño y del adolescente. Organización Mundial de la Salud. Recuperado de https://www.who.int/maternal_child_adolescent/topics/adolescence/dev/es/

Rathi, N. \& Rastogi, R. (2007). Meaning in life and psychological well-being in pre-adolescents and adolescents. Journal of the Indian Academy of Applied Psychology, 33(1), 31-38.

Rodríguez, M., Couto, M., \& Díaz, N. (2015). Modelo salutogénico: enfoque positivo de la salud. Una revisión de la literatura. Acta Odontológica Venezolana, 53(3). Recuperado de http://www.actaodontologica.com/ediciones/2015/3/art-19/

Rosenberg, M. (1965). Society and the adolescent self-image. Princenton, NJ: Princenton University Press. https://doi.org/10.1515/9781400876136

Ruini, C., Ottolini, F., Tomba, E., Belaise, C., Albieri, E., Visani, D., ... \& Fava, G. A. (2009). School intervention for promoting psychological well-being in adolescence. Journal of behavior therapy and experimental psychiatry, 40(4), 522-532. https://doi.org/10.1016/j.jbtep.2009.07.002

Ryff, C. D. (1989). Happiness is everything, or is it? Explorations on the meaning of psychological wellbeing. Journal of Personality and Social Psychology, 57(6), 1069-1081. https://doi.org/10.1037/00223514.57.6.1069

Sagone, E. \& De Caroli, M. E. (2014). Relationships between psychological well-being and resilience in middle and late adolescents. Procedia-Social and Behavioral Sciences, 141, 881-887. https://doi.org/10.1016/j.sbspro.2014.05.154

Sanders, R. A. (2013). Adolescent psychosocial, social, and cognitive development. Pediatrics in Review, 34(8), 354-358. https://doi.org/10.1542/ pir.34-8-354

Sharp, C. \& Wall, K. (2018). Personality pathology grows up: adolescence as a sensitive period. Current opinion in psychology, 21,111-116. https://doi.org/10.1016/j.copsyc.2017.11.010

Somerville, L. H., Jones, R. M., \& Casey, B. J. (2010). A time of change: behavioral and neural correlates of adolescent sensitivity to appetitive and aversive environmental cues. Brain and cognition, 72(1), 124133. https://doi.org/10.1016/j.bandc.2009.07.003

Steinberg, L. (2008). A social neuroscience perspective on adolescent risk-taking. Developmental review, 28(1), 78-106. https://doi.org/10.1016/j.dr.2007.08.002

Tabachnick, B. G. \& Fidell, L. S. (1996). Using multivariate statistics. Boston: Pearson.

Talabera, D. Y., Salgado, F. A. E., Hernández, G. B de la C., \& Borroto, P. M. (2014). Orientaciones metodológicas para el proceso formativo del médico con enfoque salutogénico. Multiciencias, 14(4), 418-424.

Thurstone, L. L. (1947). Multiple factor analysis. Chicago: University of Chicago Press.

van Dierendonck, D. (2005). The construct validity of Ryff's scale of psychological wellbeing and its extension with spiritual well-being. Personality and Individual Differences, 36(3), 629-644. https://doi.org/10.1016/S0191-8869(03)00122-3

Vázquez, C., Hervás, G., Rahona, J. J., \& Gómez, D. (2009). Bienestar psicológico y salud: Aportaciones desde la Psicología Positiva. Anuario de Psicología Clínica y de la Salud, 5(1), 15-28.

Winefield, H. R., Gill, T. K., Taylor, A. W., \& Pilkington, R. M. (2012). Psychological well-being and psychological distress: is it necessary to measure both? Psychology of Well-Being: Theory, Research and Practice, 2(1), 3. https://doi.org/10.1186/2211-1522-2-3

Recibido 17-05-2019 | Aceptado 20-09-2019

Este trabajo se encuentra bajo una Licencia Creative Commons Atribución 4.0 Internacional que permite a terceros utilizar lo publicado siempre que se dé el crédito pertinente a los autores y a Psicodebate 